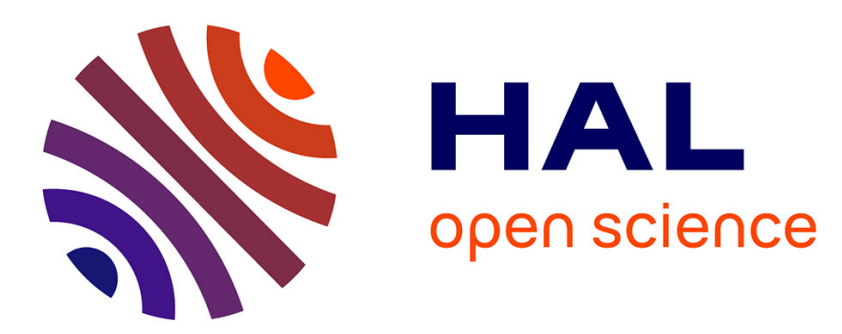

\title{
Le vieillissement de la population va-t-il entraîner une explosion des dépenses de santé?
}

\author{
Hélène Huber
}

\section{To cite this version:}

Hélène Huber. Le vieillissement de la population va-t-il entraîner une explosion des dépenses de santé?. Au chevet de la santé, La Découverte, pp.44-49, 2009, Regards croisés sur l'économie. halshs00646146

\section{HAL Id: halshs-00646146 \\ https://shs.hal.science/halshs-00646146}

Submitted on 29 Nov 2011

HAL is a multi-disciplinary open access archive for the deposit and dissemination of scientific research documents, whether they are published or not. The documents may come from teaching and research institutions in France or abroad, or from public or private research centers.
L'archive ouverte pluridisciplinaire HAL, est destinée au dépôt et à la diffusion de documents scientifiques de niveau recherche, publiés ou non, émanant des établissements d'enseignement et de recherche français ou étrangers, des laboratoires publics ou privés. 


\title{
Le vieillissement de la population va-t-il entraîner une explosion des dépenses de santé ?
}

Hélène HUBER, maître de conférences en économie à l'université Paris-1 PanthéonSorbonne.

\begin{abstract}
Le vieillissement contribue bien à l'augmentation des dépenses de santé, mais bien en deçà d'autres facteurs comme le progrès technique.
\end{abstract}

La hausse des dépenses de santé est souvent présentée comme un mal à combattre, comme un fardeau insoutenable mettant en danger la pérennité de notre système de

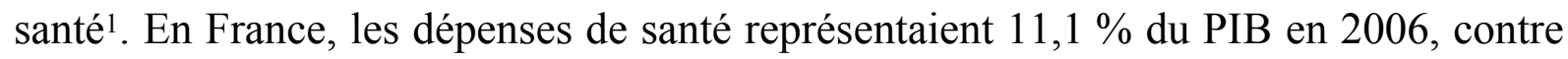
$3,8 \%$ en $1960^{2}$. Le vieillissement de la population est largement évoqué comme étant la cause principale de cette augmentation, et ceci par un raisonnement tout à fait recevable : comme ce sont les personnes les plus âgées qui consomment le plus de soins, une augmentation de leur proportion dans la population ne peut que se traduire par une augmentation des dépenses de santé. Il suffit pour s'en convaincre de jeter un œil au graphique suivant, présentant la dépense moyenne (consultations, pharmacie et hôpital, hors dépenses de dépendance) par tranche d’âge de 10 ans en France (graphique 1).

Graphique 1 - Dépense de santé moyenne par âge en 2000

\footnotetext{
1 Cet article est largement inspiré de l'excellent ouvrage de Brigitte Dormont, Les dépenses de santé - Une augmentation salutaire ?, paru en 2009 dans la collection des opuscules du Cepremap aux éditions Rue d'Ulm, ainsi que des travaux effectués lors de ma thèse de doctorat.

2 À titre de comparaison, les chiffres pour les mêmes dates aux États-Unis sont respectivement de 15,3\% et $5,1 \%$.
} 


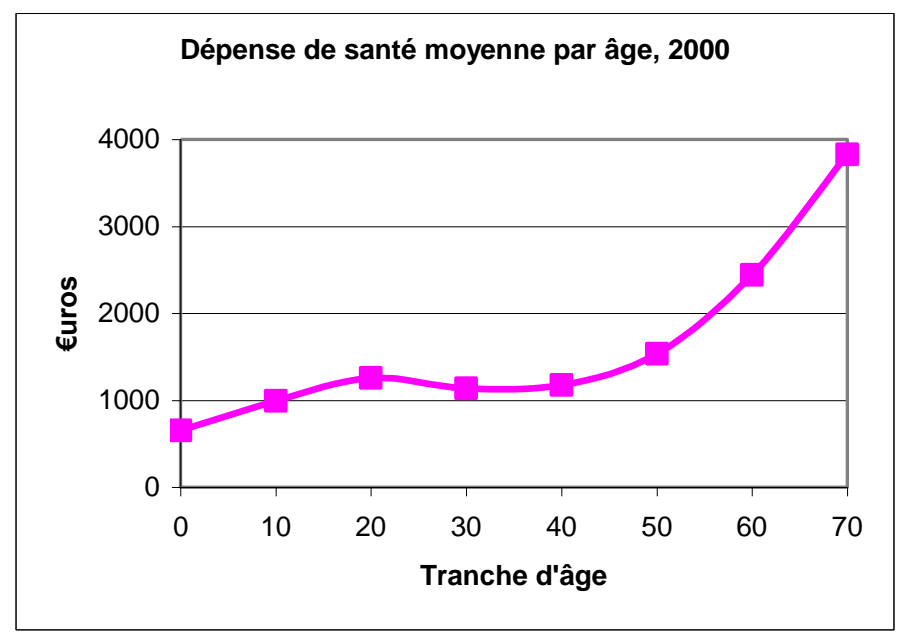

Source : Dormont, Grignon et Huber [2006], données Irdes ${ }^{3}$.

Sachant que la proportion des personnes âgées de plus de 65 ans dans la population, actuellement de $16 \%$, est selon les projections de l'Insee destinée à atteindre $26 \%$ en 2050, les inquiétudes paraissent légitimes. Elles se doublent de doutes sur la nécessité des dépenses engagées, car les personnes en fin de vie pourraient ne pas représenter un « retour sur investissement » suffisant au regard des dépenses encourues. Il suffit de se pencher sur la littérature d'économie de la santé portant sur la question de la proximité de la mort pour constater ce que l'on soupçonne déjà, qui est que les personnes en fin de vie consomment bien plus de soins que les autres. Analysant les données américaines de Medicare ${ }^{4}$, Lubitz et Riley [1993] montrent que les personnes décédant dans l'année d'observation représentent $5 \%$ des effectifs mais $30 \%$ des dépenses. Alors, le vieillissement est-il le principal moteur de la hausse des dépenses ? Et celles-ci «valent »-elles la peine d'être engagées?

\section{Décomposer les causes de la hausse des dépenses de santé}

Si le profil de dépenses par âge présenté dans le graphique précédent demeurait stable à travers les années, seules les évolutions purement démographiques causeraient

\footnotetext{
${ }^{3}$ Institut de Recherche et de Documentation en Economie de la Santé, Paris, www.irdes.fr

${ }^{4}$ Système d'assurance santé destiné aux personnes âgées de plus de 65 ans et géré par l'État américain.
} 
une variation de la dépense de santé totale, à travers un effet masse (l'évolution numérique de la population totale) et un effet de structure (la déformation de la pyramide des âges). Or, le profil de dépenses par âge n'est évidemment pas constant : le graphique 2 présente ce profil en 1992 et en 2000.

Graphique 2 - Dépenses de santé moyenne par âge en 1992 et 2000

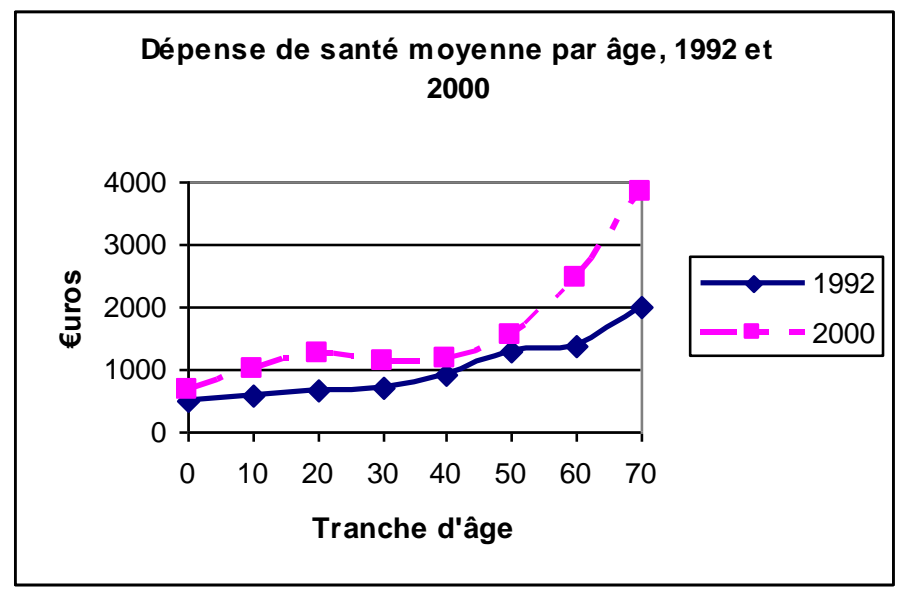

Source : Dormont, Grignon et Huber [2006], données Irdes.

Les dépenses moyennes par tranche d'âge ont augmenté, et ceci quelle que soit la tranche d'âge considérée. Plus précisément, entre 1992 et 2000, les dépenses de santé ont augmenté de $54 \%$. Sur ces $54 \%$, seuls 6 points sont dus au changement démographique (avec une importance égale de l'effet masse et de l'effet de structure) : tout le reste est attribuable à la déformation du profil de dépense par âge [Dormont, Grignon et Huber, 2006].

Or, si seules les dépenses de fin de vie étaient à l'origine de la croissance des dépenses de santé, et compte tenu du fait que la fin de vie survient de plus en plus tard, nous n'aurions pas du tout observé la déformation du profil de dépense par âge constatée. Comment, dès lors, l'expliquer ? Deux hypothèses peuvent être formulées : soit l'état de santé de la population s'est dégradé, soit les pratiques médicales ont évolué. Par le biais de micro-simulations, Dormont, Grignon et Huber [2006] décomposent finement l'augmentation de $54 \%$ des dépenses constatées. Les changements démogra- 
phiques, comme on l'a vu, ont fait augmenter les dépenses de 6 points $(+3$ pour le vieillissement et +3 pour l'augmentation de la population); les changements d'état de santé les ont fait baisser de 10 points (l'état de santé s'est amélioré) et les changements de pratique médicale, à état de santé donné, les ont fait augmenter de 58 points de pourcentage. Les changements de pratiques ont donc une importance considérable dans l'augmentation des dépenses de santé. Ces changements sont largement liés au progrès technique et à sa diffusion. Par exemple, l'opération de la cataracte est devenue courante; elle ne nécessite plus de passer une nuit à l'hôpital, et coûte donc moins cher qu'auparavant. Les personnes les plus âgées et les plus fragiles peuvent ainsi en bénéficier, ce qui augmente mécaniquement le coût moyen des soins par tranche d'âge. On constate également que l'amélioration globale de l'état de santé (-10) fait plus que compenser l'effet du vieillissement structurel de la population $(+3)$. La population semble donc « rajeunir »! Le vieillissement contribue bien à l'augmentation des dépenses de santé, mais bien en deçà d'autres facteurs comme le progrès technique.

Une nuance doit toutefois être apportée à ces résultats : les chiffres que nous étudions concernent les dépenses dites «acute » (consultations, pharmacie, hôpital) soumises à remboursement. N'y figurent donc pas les soins liés à la dépendance, qui sont évidemment amenés à croître, mais sur lesquels il n'existe malheureusement que peu de données exploitables actuellement.

L'amélioration constatée de l'état de santé entre 1992 et 2000 est en partie due aux dépenses de santé elles-mêmes. Celles-ci ne sont pas qu'un fardeau, mais un gain qu'il convient de mesurer.

\section{Évaluer les gains que permet l'augmentation des dépenses}

Les dépenses réalisées pour le maintien en vie et pour l'amélioration de la qualité de la vie des personnes ne l'ont pas été en pure perte! Les gains continuels d'espérance de vie, d'espérance de vie sans incapacité et d'espérance de vie à 60 ans peuvent en témoigner. Selon l'Insee, en 2007, l'espérance de vie à la naissance était de 77,5 ans pour les hommes et de 84,4 ans pour les femmes. En 1950, ces chiffres étaient respec- 
tivement de 63,4 et 69,2 ans. En 2007, l'espérance de vie à 60 ans était respectivement de 22,0 (hommes) et 26,9 ans (femmes). L'espérance de vie sans incapacité (ou en bonne santé) était respectivement de 62,0 (hommes) et 64,3 ans (femmes). Mais ces gains d'espérance de vie, dont chacun se réjouit, «valent »-ils les dépenses qui leur ont été consacrées ?

Pour mener une telle analyse coût-bénéfice, il faut pouvoir transcrire les gains en espérance de vie dans une métrique monétaire. C'est l'objet de la quantification en termes de «valeur statistique de la vie» (value of a statistical life, ou VSL). De nombreux malentendus existent sur cette mesure, qu'on qualifie trop rapidement de « prix de la vie ». Il ne s'agit certainement pas de calculer la valeur productive d'un individu afin de comparer les frais médicaux engagés pour sa survie avec sa production économique en cas de survie : la vie a bien évidemment une valeur en dehors de toute considération productiviste. La notion de VSL vient en fait de la planification des travaux d'équipement: lors d'une enquête, on peut demander à un échantillon d'individus combien ils seraient disposés à payer pour réduire la mortalité à une intersection routière de $\mathrm{x} \%$, par exemple grâce à la construction d'un rond-point. Si 1000 individus déclarent qu'ils sont en moyenne prêts à payer $30 €$ pour une réduction de la mortalité de $0,1 \%$, c'est-à-dire (indirectement) pour sauver la vie de l'un d'entre eux, alors la VSL ou « valeur » de cette vie statistique sauvée est égale à 1000 fois 30, c'est-à-dire $30000 €$. Les estimations de la VSL varient entre 2 et 9 millions de dollars pour les pays développés, et une valeur de 100000 dollars pour une année de vie sauvée est couramment admise. La VSL est ainsi une façon de « valoriser la vie » au niveau collectif, évidemment sans mention de l'identité de l'hypothétique personne à sauver. Nul doute que la propension individuelle à payer pour la sauvegarde de sa propre vie soit virtuellement infinie.

Pour mener à bien l'analyse coût-bénéfice qui nous intéresse, il faut ajouter à la « valeur statistique de la vie » une appréciation de la qualité de la vie : c'est l'objet des quality adjusted life years, qui associent une pondération aux années de vie. Une année de vie en bonne santé « vaut» plus qu'une année de vie en mauvaise santé. Cutler et McClellan [2001] montrent alors qu'une opération de la cataracte se traduit, en termes de quality adjusted life years, par un bénéfice d'en moyenne 95000 \$, alors qu'elle 
coûte 3000 \$. Le gain net de cette opération, majoritairement destinée aux personnes âgées, est donc manifeste.

La valorisation de la vie statistique combinée à la prise en compte de la qualité permet donc d'apprécier les gains liés aux dépenses de santé. Murphy et Topel [2006] estiment ainsi qu'entre 1970 et 2000 aux États-Unis, les gains en espérance de vie auraient en moyenne chaque année ajouté à la richesse nationale une valeur équivalente à $50 \%$ du PIB, à comparer à la part du PIB consacrée aux dépenses de santé (en croissance constante et égale à $15 \%$ pour les États-Unis en 2000).

Les quelques travaux présentés ici montrent qu'au moins pour ce qui est des soins dits « acute », il est vraisemblable que le vieillissement de la population ne provoque pas une explosion des dépenses. L'augmentation constatée serait plutôt à mettre sur le compte des changements de pratiques à état de santé donné, et le vieillissement démographique en tant que tel n'aurait qu'un impact mineur. Les gains en termes de longévité et de qualité de vie que permettent les dépenses de santé paraissent de plus largement couvrir leur coût.

\section{BIBLIOGRAPHIE}

DORMONT B. (2009), Les dépenses de santé - Une augmentation salutaire ?, éditions Rue d'Ulm, collection des opuscules du Cepremap, Paris.

DORMONT B., GRIGNON M. et HuBER H. (2006), « Health expenditures growth : reassessing the threat of aging », Health Economics.

HuBER H. (2006), « Vieillissement, dépenses de santé et inégalités de recours aux soins : essais de micro-économétrie appliquée », Thèse de doctorat en Sciences Economiques, Université Paris 10 Nanterre.

LUBITZ J.B. et RILEY G.F. (1993), « Trends in Medicare payments in the last years of life », New England Journal of Medicine.

MurPhy K. et TOPEL R.H. (2006), « The value of life and longevity », Journal of Political Economy. 
